\title{
Primary Cardiac Lymphoma: Three Case Reports and a Review of the Literature
}

\author{
Clifford Michael Csizmar¹, Zohar Sachs², Zuzan Cayci ${ }^{3}$, Lihong Bu${ }^{4}$, Michael Andrew Linden \\ ${ }^{1}$ School of Medicine, University of Minnesota, Minneapolis, USA \\ ${ }^{2}$ Division of Hematology, Oncology, and Transplantation, Department of Medicine, University of Minnesota, Minneapolis, USA \\ ${ }^{3}$ Department of Radiology, University of Minnesota, Minneapolis, USA \\ ${ }^{4}$ Division of Hematopathology, Department of Laboratory Medicine and Pathology, University of Minnesota, Minneapolis, USA \\ Email: linde013@umn.edu
}

How to cite this paper: Csizmar, C.M., Sachs, Z., Cayci, Z., Bu, L. and Linden, M.A. (2021) Primary Cardiac Lymphoma: Three Case Reports and a Review of the Literature. Open Journal of Blood Diseases, 11, 120-132.

https://doi.org/10.4236/ojbd.2021.114012

Received: October 1, 2021

Accepted: November 29, 2021

Published: December 2, 2021

Copyright $\odot 2021$ by author(s) and Scientific Research Publishing Inc. This work is licensed under the Creative Commons Attribution International License (CC BY 4.0).

http://creativecommons.org/licenses/by/4.0/

(c) (i) Open Access

\begin{abstract}
Primary cardiac lymphoma (PCL) is a rare entity that comprises only $1 \%$ $2 \%$ of all cardiac tumors. Due to their scarcity and variable clinical presentation, early diagnosis is challenging. In this series, three cases of PCL from a single institution are described, which highlight the spectrum of presenting features and emphasize common principles. In the first case, a 73-year-old male who presented with dyspnea was found to have a $12.1 \mathrm{~cm}$ mass in the right ventricle. Biopsy via cardiac catheterization revealed diffuse large B cell lymphoma (DLBCL). He was treated with chemoimmunotherapy and survived for two months. The second case describes a 55-year-old female who presented with chest pain. Imaging revealed a $3.1 \mathrm{~cm}$ right atrial mass and bilateral pleural effusions, with cytology from the latter demonstrating DLBCL. She was lost to follow up after three cycles of chemoimmunotherapy. In the last case, an 80-year-old female presented with weakness. A $4.0 \mathrm{~cm}$ mass was discovered in the right atrium and the patient expired shortly after admission. Autopsy confirmed the diagnosis of DLBCL. These case summaries are followed by a review of the clinical presentation, diagnostic approach, and treatment outcomes of PCL.
\end{abstract}

\section{Keywords}

Primary Cardiac Lymphoma, Diffuse Large B Cell Lymphoma, Cardiac Tumors

\section{Introduction}

Primary cardiac lymphoma (PCL) is a rare entity that accounts for up to $2 \%$ of all primary cardiac tumors [1] [2]. The majority of PCLs are B cell malignancies, 
with diffuse large B cell lymphoma (DLBCL) being the most common histologic subtype; however, other hematologic neoplasms have been infrequently described [3] [4] [5]. The clinical presentation of PCL is widely varied, and one third of patients have symptoms of congestive heart failure (CHF) [3]. Due to the rarity of PCL, diagnosis can be challenging and patients often undergo multiple imaging modalities in order to distinguish PCL from the more common atrial myxomas and thrombi [6] [7] [8]. Ultimately, tissue biopsy is required to establish the diagnosis and provide classification of the lymphoma. In many instances, such tissue sampling is performed at the time of open surgical resection or debulking [9] [10] [11]. Beyond surgery, chemoimmunotherapy using a first-line regimen appropriate for the lymphoma subtype is the standard of care.

Here, we describe three cases of PCL that presented to our institution over the past several years (Table 1). All malignancies arose in immunocompetent patients

Table 1. Summary of three primary cardiac diffuse large B cell lymphoma cases.

\begin{tabular}{|c|c|c|c|}
\hline & Case 1 & Case 2 & Case 3 \\
\hline Age (years) & 73 & 55 & 80 \\
\hline Sex & Male & Female & Female \\
\hline Presentation & $\begin{array}{c}\text { Dyspnea } \\
\text { Peripheral edema }\end{array}$ & $\begin{array}{c}\text { Dyspnea } \\
\text { Substernal chest pain }\end{array}$ & $\begin{array}{c}\text { Dyspnea } \\
\text { Weakness }\end{array}$ \\
\hline ECG & $\begin{array}{l}\text { Right bundle } \\
\text { branch block } \\
\text { Prolonged QTc }\end{array}$ & $\begin{array}{c}\text { First-degree } \\
\text { AV block } \\
\text { Prolonged QTc }\end{array}$ & $\begin{array}{c}\text { Atrial fibrillation } \\
\text { Prolonged QTc }\end{array}$ \\
\hline Echocardiogram & $\begin{array}{l}12.1 \mathrm{~cm} \text { mass } \\
\text { in the RV }\end{array}$ & $\begin{array}{l}3.1 \mathrm{~cm} \text { mass } \\
\text { in the RA }\end{array}$ & $\begin{array}{l}4.0 \mathrm{~cm} \text { mass } \\
\text { in the RA }\end{array}$ \\
\hline Other Imaging & $\begin{array}{l}\text { Cardiac MRI } \\
\text { CT C/A/P }\end{array}$ & $\begin{array}{c}\text { Cardiac MRI } \\
\text { CT C/A/P } \\
\text { PET/CT }\end{array}$ & CT Chest \\
\hline HIV Status & Negative & Negative & Not assessed \\
\hline EBV Serology & Not assessed & Positive IgG & Not assessed \\
\hline Tissue Sampling & Biopsy & Biopsy & Autopsy \\
\hline Diagnosis & DLBCL & DLBCL & DLBCL \\
\hline Treatment & $\mathrm{R}-\mathrm{COEP} \times 2$ & $\begin{array}{c}\mathrm{R}-\mathrm{CVP} \times 1 \\
\mathrm{R}-\mathrm{CHOP} \times 2\end{array}$ & None \\
\hline Survival & 2 months & Lost to follow-up & 1 week \\
\hline
\end{tabular}

Abbreviations: ECG, electrocardiogram; AV, atrioventricular; RV, right ventricle; RA, right atrium; MRI, magnetic resonance imaging; CT, computed tomography; C/A/P, chest, abdomen, and pelvis; PET, positron emission tomography; EBV, Epstein-Barr virus; HIV, human immunodeficiency virus; IgG, immunoglobulin G; DLBCL, diffuse large B cell lymphoma; R-COEP, rituximab, cyclophosphamide, vincristine, etoposide, and prednisone; R-CVP, rituximab, cyclophosphamide, vincristine, and prednisone; R-CHOP, rituximab, cyclophosphamide, doxorubicin, vincristine, and prednisone. 
aged 55 - 80 years and presented as right-sided cardiac masses. All cases were diagnosed as DLBCL, and two of the three neoplasms expressed CD5. These cases highlight the broad spectrum of presenting symptoms, pathologic features, and treatment outcomes associated with PCL.

\section{Case Presentations}

\subsection{Case 1}

A 73-year-old male presented with six months of progressive dyspnea and lower extremity edema refractory to diuretics. Fevers, night sweats, and weight loss were denied. An electrocardiogram (ECG) revealed a right bundle branch block with a prolonged QTc interval (487 ms). A subsequent echocardiogram showed a large, infiltrating, lobular, heterogenous mass in the right ventricle extending into pericardial space. Accordingly, the right ventricle was moderately dilated with decreased systolic function, though the left ventricular ejection fraction (LVEF) was preserved (55\% - 60\%).

Cardiac magnetic resonance imaging (MRI) demonstrated a $6.2 \times 8.5 \times 12.1$ $\mathrm{cm}$ mass within the right ventricle infiltrating the free wall and extending into the pericardial space (Figure 1(A)). The right papillary muscle, right ventricular outflow tract, pulmonic valve, and right side of the interventricular septum were similarly involved. Two smaller masses were observed at the posterior aspect of the mitral annulus $(1.4 \times 2.0 \mathrm{~cm})$ and adjacent to the ascending aorta $(1.6 \times 2.1$ $\mathrm{cm}$ ); these were similar in consistency to the right ventricular mass and felt to be metastases. Computed tomography (CT) imaging of the chest, abdomen, and pelvis recapitulated the cardiac mass (Figure 1(B)), but did not demonstrate any extracardiac tumors or suspicious lymphadenopathy. MRI of the brain did not reveal any intracranial pathology.

The right ventricular mass was biopsied via cardiac catheterization. Histology showed diffuse infiltration of large malignant lymphoid cells (Figure 1(C)). Immunohistochemical (IHC) staining revealed an unusual phenotype of CD20+, CD10+, CD5+, and CD23-cells, consistent with DLBCL, germinal center subtype [12]. An in situ hybridization study for EBV (EBER) was negative. Flow cytometry
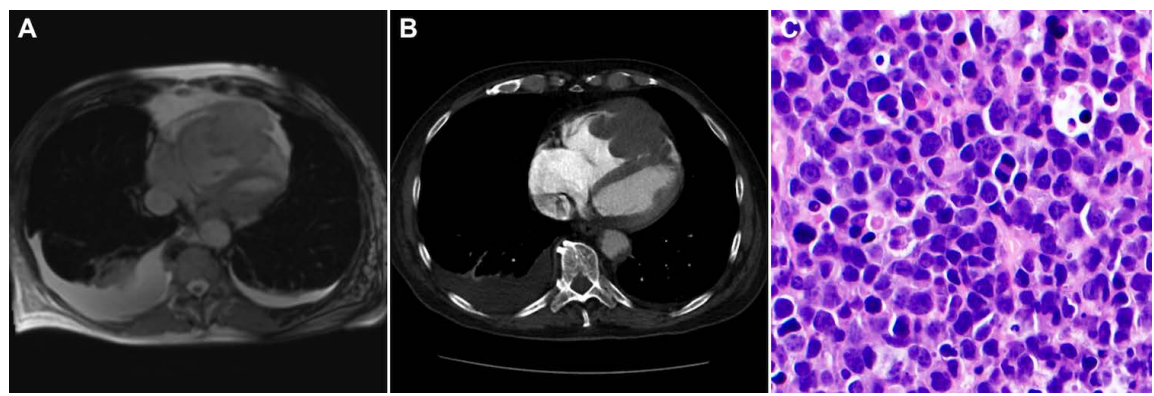

Figure 1. Cardiac imaging and histology for case one. Cardiac MRI (A) and chest CT (B) imaging depicting a large right ventricular mass. (C) Hematoxylin and eosin (H \& E) staining of biopsied tissue from cardiac mass depicting diffuse infiltration of large, malignant, lymphoid cells; 500× magnification. 
confirmed the phenotype, and fluorescence in situ hybridization (FISH) assays were negative for rearrangements in $B C L 1, B C L 2, B C L 6$, or $M Y C$; however, abnormal gains of 3q, 8q, 11q, 14q, and 18q were identified. Thus, a diagnosis of de novo CD5+ primary cardiac DLBCL was made.

The patient was started on chemotherapy with rituximab, cyclophosphamide, vincristine, etoposide, and prednisone (R-COEP). He received two cycles before expiring from cardiogenic shock two months after diagnosis.

\subsection{Case 2}

A 55-year-old female presented with two months of progressive dyspnea, orthopnea, lower extremity edema, and dull chest ache. She denied fevers, night sweats, and weight loss. An ECG showed a first-degree atrioventricular (AV) block with a prolonged QTc interval (497 ms), and an echocardiogram identified a right atrial mass. CT of the chest, abdomen, and pelvis demonstrated a large mass within the right atrium and ventricle with bilateral pleural effusions; cardiac MRI further revealed severely limited blood flow through the right heart. Combined positron emission tomography with CT (PET/CT) showed a hypermetabolic mass centered in the right atrium abutting the aorta with subsequent narrowing of the superior vena cava (Figure 2(A) and Figure 2(B)). No other masses, lymphadenopathy, or foci of hypermetabolism were seen.

Pleural effusion cytology showed a population of large anaplastic cells with irregularly-shaped nuclei, high nucleocytoplasmic ratios, and scant vacuolated cytoplasm. IHC demonstrated that the cells were positive for CD20, PAX5, CD5, and MUM1 with a very high proliferation rate by Ki67 staining (>90\%). Stains for Bcl2, Bcl6, CD10, CD30, CD56, CD138, ALK1, cyclin D1, EMA, HHV8, and EBER-ISH were all negative. Flow cytometry confirmed expression of CD5, CD19, CD20, partial CD22, CD38, CD45, and kappa monotypic surface immunoglobulin light chains; they lacked CD10, CD14, CD56, and CD138. Collectively, these results were diagnostic of DLBCL, and have been previously reported in greater detail [13].

To confirm the cardiac origin of the pleural fluid findings, the patient underwent endomyocardial biopsy via right heart catheterization. Histology showed diffuse infiltration by large lymphoid cells with extensive necrosis (Figure 2(C)). The cells expressed CD20, CD45, and CD99 by IHC staining, but lacked CD3, myogenin, and cytokeratins AE1/AE3 (Figures 2(D)-(F)), confirming de novo CD5+ primary cardiac DLBCL.

The patient was treated with a modified regimen of rituximab, cyclophosphamide, doxorubicin, vincristine, and prednisone (R-CHOP) with quick improvement of her symptoms. Doxorubicin was omitted from the first cycle due to concerns over possible rupture of the right ventricular free wall. She received two additional cycles as usual without complications, and an echocardiogram after cycle three showed no evidence of residual tumor. She then transferred her care to a facility closer to her residence and was lost to follow up. 


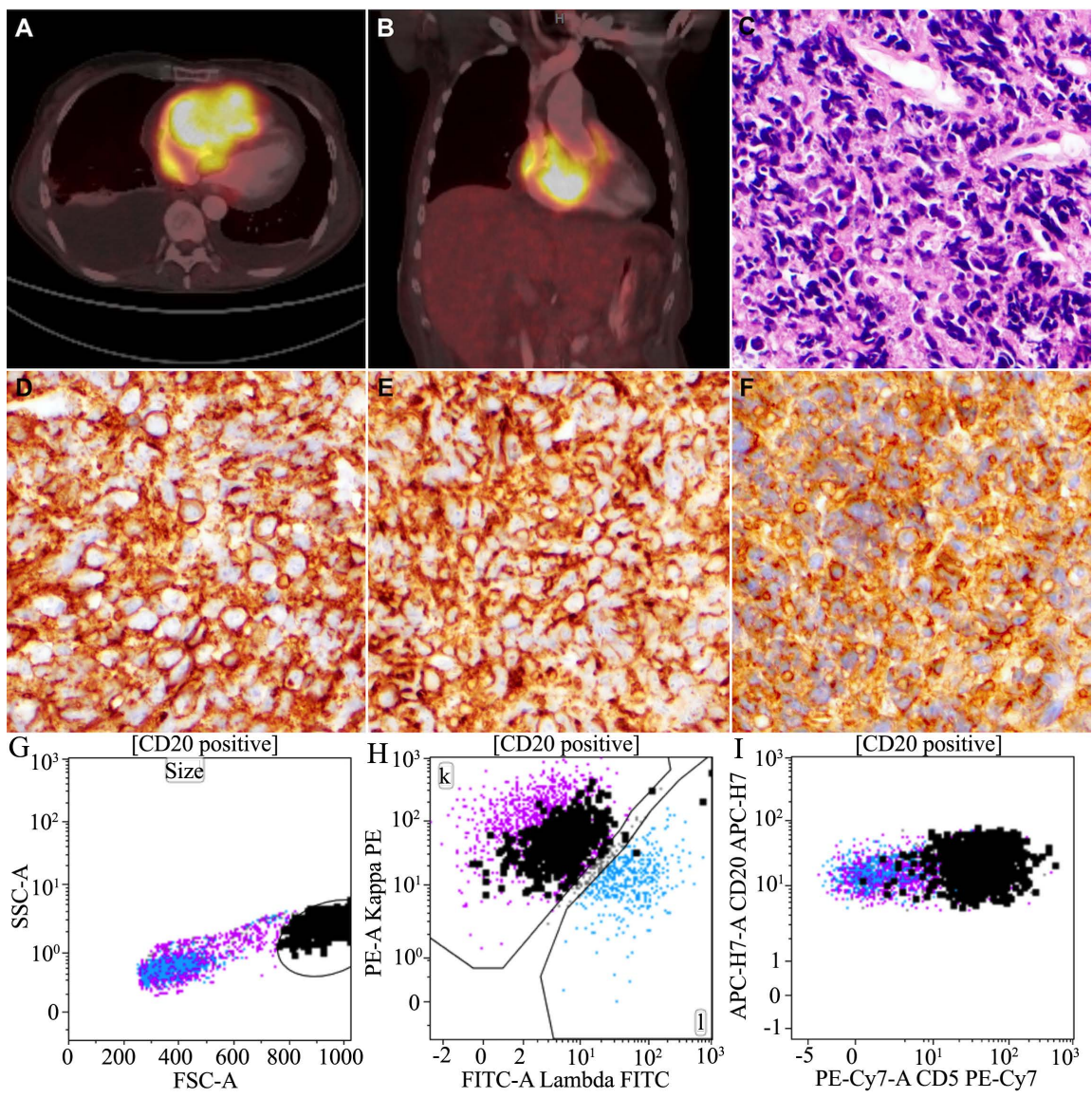

Figure 2. Cardiac imaging, histology, and flow cytometry for case two. (A) Axial and (B) coronal sections of PET/CT imaging depicting a large hypermetabolic mass centered in the right atrium with involvement of the right ventricle. (C) $\mathrm{H} \& \mathrm{E}$ staining of biopsied tissue from cardiac mass depicting diffuse infiltration by large lymphoid cells with extensive necrosis. IHC staining of the mass demonstrating expression of (D) CD20, (D) CD45, and (F) CD99. Histologic images all depicted at 500× magnification. ((G)-(I)) Flow cytometry of pleural fluid. The three gated dotplots show all B cells that coexpress CD19 and CD20. The population of interest is gated in black, with non-neoplastic B cells in magenta and aqua. Large cells are gated based on size (high FSC) in panel (G). They are kappa monotypic (panel (H)), and they express CD20 and dim CD5 (panel (I)).

\subsection{Case 3}

An 80-year-old female presented to the emergency department with two days of progressive weakness, hypotension, and dyspnea. She denied fevers, night sweats, and weight loss. She was mildly tachypneic and hypoxic on exam, and ECG showed atrial fibrillation with a rapid ventricular rate and a prolonged QTc interval $(472 \mathrm{~ms})$. A urinalysis was positive for bacteria and her lactate was elevated. Broad spectrum antibiotics were started for sepsis. Due to persistent hypoxia, CT of the chest was obtained to evaluate for pulmonary emboli. While small emboli were noted, there was also cardiomegaly with a moderate-sized pericardial effusion (Figure $3(\mathrm{~A})$ ). An echocardiogram revealed a mobile, pedunculated right atrial mass $(4.0 \times 3.6 \mathrm{~cm})$ prolapsing in and out of the tricuspid valve. The right ventricle was severely dilated with preserved LVEF (65\% - 70\%). 


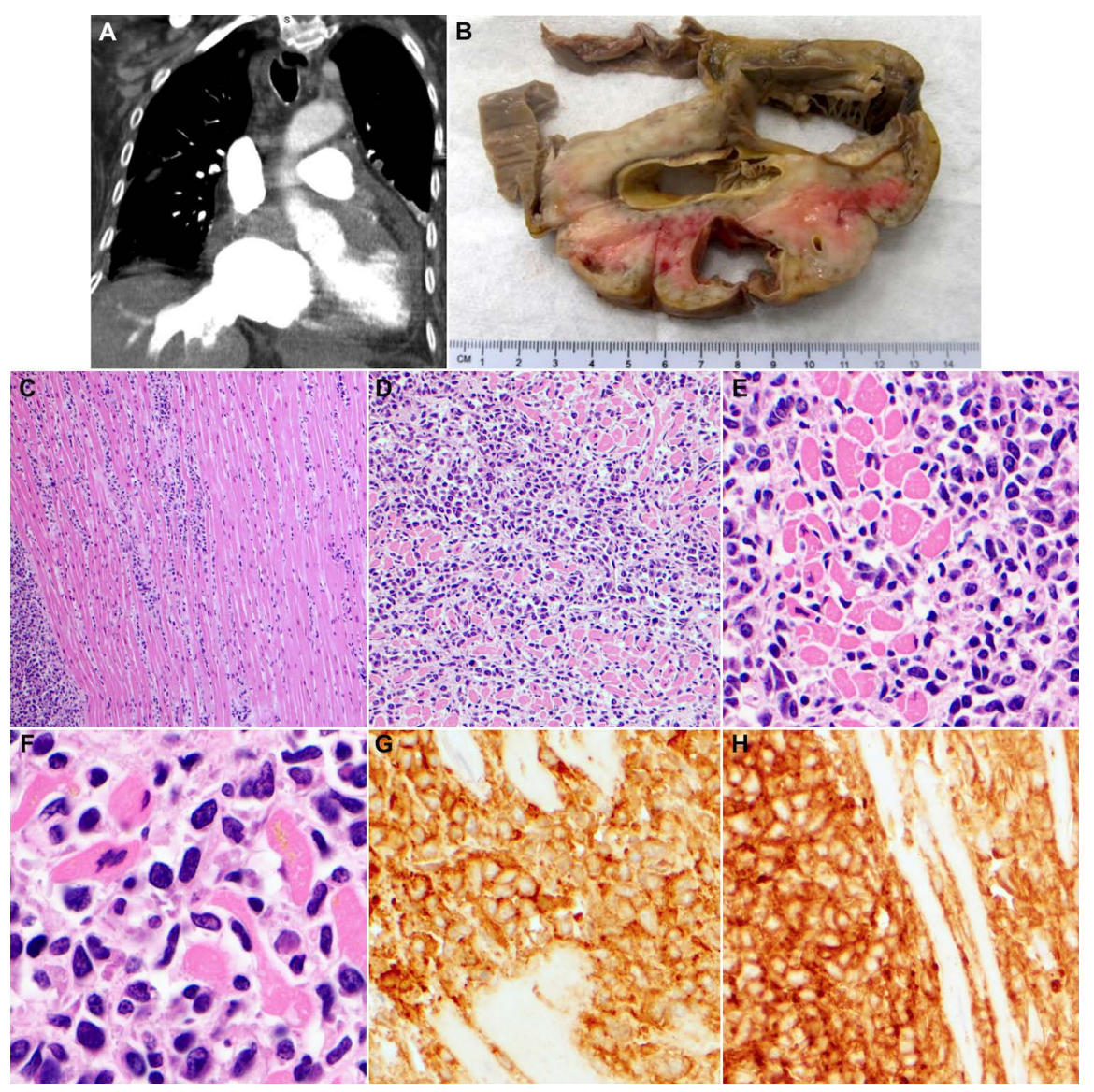

Figure 3. Cardiac imaging, autopsy findings, and histology for case three. (A) Coronal section of chest CT imaging depicting an enlarged cardiac silhouette with irregular thickening of the right atrium and ventricle. (B) Gross cross-section of the cardiac mass demonstrating invasion into the myometrium and encasement of the aorta and pulmonic trunk. $\mathrm{H} \& \mathrm{E}$ staining of biopsied tissue from cardiac mass depicting large lymphoid cells dissecting the myocardial fascicles at (C) $100 \times$, (D) $200 \times$, (E) $500 \times$, and (F) $1000 \times$ magnification. IHC staining of the mass demonstrating expression of (G) CD20 and (H) CD45, both at $500 \times$ magnification.

The patient's clinical condition deteriorated rapidly and she was transferred to the intensive care unit with multi-organ failure. Cardiothoracic surgery was consulted but, due to her condition, she was not deemed a surgical candidate. Consistent with the patient's wishes, comfort cares were pursued and she expired shortly thereafter.

The husband consented to autopsy, which revealed a cardiac tumor measuring $12.8 \times 12.5 \mathrm{~cm}$ with thickness up to $2.5 \mathrm{~cm}$ arising from the wall of right atrium and right ventricle, extending to the interventricular septum, left atrium and left ventricle (Figure $3(\mathrm{~B})$ ). A $4.2 \times 3.5 \times 3.5 \mathrm{~cm}$ pedunculated mass was found in the right atrium and continued to extend into the right ventricle. The right ventricular septum was focally involved. The right atrial and ventricular chamber volumes were significantly decreased, and the tricuspid and pulmonary valves were compressed by the tumor. The tricuspid valve leaflets were also focally involved and displaced by the tumor. The endocardial surfaces showed polypoid 
tumor bulging in the right atrium and ventricle. The myocardium was extensively involved or replaced by tumor, as were the atrial and ventricular septa. Multifocal areas of tumor necrosis were also identified.

Histologically, tumor cells were identified on all cardiac sections and appeared dyscohesive with moderate cytoplasm and large pleomorphic nuclei. They infiltrated the myocytes and dissected the myocardial fascicles in all involved regions (Figures 3(C)-(F)). By IHC, the neoplastic cells stained positive for CD20 and CD45, but negative for CD3, CD5, CD10, and cytokeratins AE1/AE3 (Figure $3(\mathrm{G})$ and Figure $3(\mathrm{H}))$. The morphology and staining pattern were consistent with DLBCL. No other areas of tumor involvement were identified on autopsy or in previous imaging, compatible with primary cardiac lymphoma.

\section{Discussion}

\subsection{Epidemiology}

Extranodal disease is observed in up to $40 \%$ of cases of non-Hodgkin lymphoma (NHL) and represents an independent negative prognostic factor [14]. Primary extranodal disease-arising de novo in a non-hematolymphoid tissue-is less common, representing approximately $20 \%$ of NHL cases and with the most common sites of involvement being the gastrointestinal tract, liver, and skin [15] [16]. Likewise, cardiac involvement by NHL occurs in roughly $20 \%$ of NHL patients who undergo autopsy [17]; however, PCL is rare and comprises only $1 \%-2 \%$ of all cardiac tumors [1] [2] (Table 2). DLBCL appears to be the most common cardiac lymphoma, representing $71 \%$ of reviewed cases [3]. Burkitt lymphoma and chronic lymphocytic leukemia/small lymphocytic lymphoma (CLL/SLL) were also frequently reported [3]. Cardiac involvement by natural killer (NK) cell [18] and Hodgkin lymphomas [17] have been described, though these appear to be incredibly rare.

\subsection{Presentation}

In modern series, PCL has been reported in patients aged $10-83$ years with a median age at the time of diagnosis ranging from 55 - 65 years [3] [4] [5]. Most patients present with cardiac complaints, including symptoms of congestive heart failure $(31 \%-78 \%)$, chest pain $(19 \%-46 \%)$, or arrhythmia $(8 \%$ 56\%) [3] [4]. Less commonly, superior vena cava (SVC) syndrome [9] [10] [19], odynophagia [20], and embolic phenomena [21] were the chief concern. Similar to other early-stage lymphomas, traditional B symptoms are uncommon, described by only $7 \%-26 \%$ of patients [3] [4]. This is reflected in the cases presented here, where none of the three patients endorsed fevers, chills, night sweats, or weight loss.

Most cases of PCL manifest with a cardiac mass. Involvement has been described in all four chambers of the heart, the interatrial and interventricular septa, and the pericardium. The right atrium is the single most common site (66\% $77 \%$ of cases), and most cases (up to 92\%) involve the right heart [4]. Indeed, all 
Table 2. Reported features of primary cardiac lymphoma.

\begin{tabular}{|c|c|c|c|}
\hline Category & Feature & Value & References \\
\hline Epidemiology & $\begin{array}{l}\text { Primary cardiac lymphoma } \\
\text { Secondary cardiac involvement }\end{array}$ & $\begin{array}{c}1 \%-2 \% \text { of all } \\
\text { cardiac tumors } \\
\text { Up to } 20 \% \\
\text { of NHL cases }\end{array}$ & [1] [2] [17] \\
\hline Age at diagnosis & $\begin{array}{l}\text { Median } \\
\text { Range }\end{array}$ & $\begin{array}{l}55-65 \text { years } \\
10-83 \text { years }\end{array}$ & [3] [4] [5] \\
\hline Sex & $\begin{array}{l}\text { Male } \\
\text { Female }\end{array}$ & $\begin{array}{l}65 \%-85 \% \\
15 \%-35 \%\end{array}$ & [3] [4] \\
\hline Presenting features & $\begin{array}{c}\text { Heart failure } \\
\text { Chest pain } \\
\text { Arrhythmia } \\
\text { Constitutional symptoms } \\
\text { SVC syndrome }\end{array}$ & $\begin{array}{l}31 \%-78 \% \\
19 \%-46 \% \\
8 \%-56 \% \\
7 \%-26 \% \\
5 \%-15 \%\end{array}$ & $\begin{array}{c}{[3][4][9]} \\
{[10][32]}\end{array}$ \\
\hline $\begin{array}{l}\text { Location and } \\
\text { involvement }\end{array}$ & $\begin{array}{c}\text { Right atrium } \\
\text { Right ventricle } \\
\text { Left atrium } \\
\text { Left ventricle } \\
\text { Septum } \\
\text { Pericardial effusion }\end{array}$ & $\begin{array}{l}66 \%-77 \% \\
25 \%-37 \% \\
12 \%-30 \% \\
2 \%-21 \% \\
2 \%-41 \% \\
30 \%-58 \%\end{array}$ & [4] [32] \\
\hline Histology & $\begin{array}{c}\text { DLBCL } \\
\text { Burkitt } \\
\text { CLL/SLL } \\
\text { T cell } \\
\text { Other or NOS }\end{array}$ & $\begin{array}{c}71 \%-92 \% \\
7 \%-8 \% \\
4 \%-8 \% \\
3 \%-5 \% \\
\text { Up to } 10 \%\end{array}$ & [3] [4] [32] \\
\hline Treatment & $\begin{array}{c}\text { Chemotherapy } \\
\text { Surgery } \\
\text { Radiation } \\
\text { Combination therapy }\end{array}$ & $\begin{array}{l}52 \%-89 \% \\
16 \%-28 \% \\
8 \%-20 \% \\
12 \%-23 \%\end{array}$ & [3] [4] [32] \\
\hline Outcomes & Median OS & $3-63$ months & [3] [4] \\
\hline
\end{tabular}

Abbreviations: NHL, non-Hodgkin lymphoma; SVC, superior vena cava; DLBCL, diffuse large B cell lymphoma; CLL, chronic lymphocytic leukemia; SLL, small lymphocytic lymphoma; NOS, not otherwise specified; OS, overall survival.

three of the cases reported here were located on the right side, and two originated in the right atrium. 


\subsection{Differential Diagnosis}

The differential for a cardiac mass includes infections, neoplasms, thrombi, and structural abnormalities [7]. Infectious etiologies include bacterial endocarditis and myocardial abscesses. In immunocompromised patients, fungal organisms are also a consideration [22] [23] [24]. Structural causes include false tendons, moderator bands, and ruptured chordae tendinae [7]. Thrombi can result from arrhythmia, hypokinesis, or represent emboli from a peripheral source. Cardiac neoplasms are more likely to represent metastases than primary tumors [25]. Benign myxomas are the most common cardiac tumor, accounting for roughly one third of cases [26]. As a class, sarcomas represent the most common primary cardiac malignancy in immunocompetent people, with angiosarcomas being the dominant subtype [7].

Interestingly, all three patients in this series had a prolonged QTc interval on their presenting ECG. This finding is not routinely associated with infectious etiologies, cardiac thrombi, or non-infiltrating masses [27]. Therefore, a prolonged QTc interval may be suggestive of a malignant process in the appropriate clinical setting.

\subsection{Diagnostic Approach}

A tissue biopsy is required to diagnose PCL and classify the lymphoma appropriately. Due to the invasive nature of cardiac tissue sampling, however, cardiac tumors are often characterized by multiple modes of imaging prior to biopsy. Most cardiac masses are first identified on a transthoracic echocardiogram (TTE). Though no formalized workflow has been developed for optimal characterization, cardiac MRI, CT, and PET imaging have all been utilized [4] [6] [13].

\subsection{Treatment}

Treatment for PCL typically follows the same recommendations as other extranodal manifestations of the same lymphoma subtype. In the case of DLBCL, for example, chemoimmunotherapy with a rituximab backbone is frequently used. In cases of "double-hit" lymphoma, complete remission (CR) has been achieved using either R-CHOP or R-CVP (cyclophosphamide, vincristine, and methylprednisolone) regimens [28] [29]. In these cases, however, subsequent hematopoietic stem cell transplantation (HSCT) was not pursued and systemic relapse was detected within eight to nine months [28] [29]. Despite evidence that higher intensity chemoimmunotherapy regimens provide more durable remissions in double-hit lymphomas [30], the use of such protocols has not been reported in PCL. This could be due to the relatively poor performance status and cardiac function of PCL patients at presentation. Likewise, HSCT has not been reported in PCL.

In a small case series of thirteen PCL patients, CR was achieved in $62 \%$ of patients using first-line chemotherapy for the respective lymphoma subtype [4]. However, no patients proceeded to HSCT and relapse was documented in 55\% of cases, mostly at extranodal sites [4].

One caveat is whether patients require surgical debulking of the cardiac mass 
prior to the initiation of chemotherapy. Urgent surgery is commonly pursued in patients who present with SVC syndrome [9] [10], rapidly progressive heart failure [11], or with atypical symptoms that obscure the diagnosis until tissue is obtained during resection [2] [21]. Otherwise, prompt initiation of chemotherapy is often sufficient to provide rapid relief of symptoms [13].

\subsection{Outcomes}

The median overall survival for patients diagnosed with PCL ranges widely from 3 - 63 months [3] [4]. Patients who present with symptoms of CHF and those who do not receive chemotherapy do poorly, with median survival rates of less than two months [3] [20]. Overall survival appears best in patients with indolent $\mathrm{B}$ cell malignancies who receive chemotherapy. In one large retrospective analysis of such patients, median survival was not reached ( $>5$ years) in in the group of patients who survived beyond one month from diagnosis [3].

In this series, one patient (case 3) presented with two days of rapidly-progressive symptoms, did not receive chemotherapy, and died three days after diagnosis of the atrial mass. The other two patients (cases $1 \& 2$ ) presented with several weeks of CHF symptoms and were treated with rituximab-containing regimens. One patient passed away two months after diagnosis, having completed only two cycles of R-COEP. The other was lost to follow up after four months, having completed one cycle of R-CVP followed by two cycles of R-CHOP with complete resolution of the cardiac tumor on echocardiogram at that time.

Notably, the two treated patients (cases $1 \& 2$ ) were both diagnosed with de novo CD5+ DLBCL. Expression of CD5 is traditionally a poor prognostic factor in DLBCL [31]. While treatment outcomes have improved since the introduction of rituximab, there is evidence to suggest that this benefit is largely restricted to CD5-negative DLBCL. In a small cohort of patients with CD5+ DLBCL treated with $(\mathrm{n}=11)$ and without $(\mathrm{n}=8)$ rituximab, the two-year overall survival of the two groups was not statistically different ( $59 \%$ and $50 \%$, respectively, $\mathrm{p}=0.70$ ) [31]. While the patient who was lost to follow up had a good initial response to chemotherapy, her presentation with CHF symptoms and CD5 positivity may portend an inferior long-term outcome.

\section{Conclusions}

Here, we report three cases of PCL that arose de novo in immunocompetent patients. All neoplasms were diagnosed as DLBCL and arose on the right side of the heart. Two were positive for CD5 expression, a feature that traditionally portends inferior outcomes. Of the three patients, two are confirmed deceased and expired shortly after diagnosis.

Ultimately, primary cardiac lymphomas remain rare malignancies that can present with a multitude of symptoms. Because of this, diagnosis of PCL can be elusive and often requires multimodal imaging prior to tissue biopsy. Chemotherapy is typically the mainstay of treatment, with the specific regimen chosen 
based on the pathologic diagnosis and patient comorbidities. Outcomes are varied, though they appear to be improving with advances in chemo- and immunotherapy. Currently, patients with indolent NHL who can tolerate chemotherapy have the longest overall survival. Future goals should include improving the ability to diagnose PCL without invasive tissue sampling and determining the role of HSCT in PCL treatment.

\section{Acknowledgements}

The authors gratefully acknowledge Kelly Miles for her assistance in preparing the autopsy specimen. This work was supported by the National Institutes of Health (T32 GM008244) and the University of Minnesota.

\section{Supporting Information}

Representative electrocardiograms (ECGs) for each patient case are available on the SCIRP website at DOI: ***.

\section{Conflicts of Interest}

The authors have no conflicts of interest associated with this work.

\section{Research Conduct}

The study proposal was submitted to the institutional review board (IRB, submission ID 00013473) and deemed exempt (not human research). All patients consented to have their de-identified data used for research purposes upon hospital admission.

\section{References}

[1] Miller, D.V., Mookadam, F., Mookadam, M., Edwards, W.D. and Macon, W.R. (2007) Primary Cardiac Plasmablastic (Diffuse Large B-Cell) Lymphoma Mimicking Left Ventricular Aneurysm with Mural Thrombus. Cardiovascular Pathology, 16, 111-114. https://doi.org/10.1016/j.carpath.2006.09.003

[2] Aimanan, K., Kumar, K.S., Mohd Arif, M.N., Noor Zuraini, M., Ramdzan, M.J. and Hamdan, L. (2016) Rare Intracardiac Tumor: Primary Cardiac Lymphoma Presenting as Atypical Angina. Case Reports in Medicine, 2016, Article ID: 4125295. https://doi.org/10.1155/2016/4125295

[3] Gordon, M.J., Danilova, O., Spurgeon, S. and Danilov, A.V. (2016) Cardiac NonHodgkin's Lymphoma: Clinical Characteristics and Trends in Survival. European Journal of Haematology, 97, 445-452. https://doi.org/10.1111/ejh.12751

[4] Carras, S., Berger, F., Chalabreysse, L., Callet-Bauchut, E., Cordier, J.F., Salles, G., et al. (2017) Primary Cardiac Lymphoma: Diagnosis, Treatment and Outcome in a Modern Series. Hematological Oncology, 35, 510-519. https://doi.org/10.1002/hon.2301

[5] Patel, J., Melly, L. and Sheppard, M.N. (2010) Primary Cardiac Lymphoma: B- and T-Cell Cases at a Specialist UK Centre. Annals of Oncology, 21, 1041-1045. https://doi.org/10.1093/annonc/mdp424

[6] Wang, S., Li, M., Zhang, L. and Xie, M. (2018) Multimodal Imaging Evaluation of a 
Primary Cardiac Lymphoma in an Immunocompetent Patient. Echocardiography, 35, 2121-2123. https://doi.org/10.1111/echo.14150

[7] Kaplan, L.D., Afridi, N.A., Holmvang, G. and Zukerberg, L.R. (2003) Case Records of the Massachusetts General Hospital. Weekly Clinicopathological Exercises. Case 31-2003. A 44-Year-Old Man with HIV Infection and a Right Atrial Mass. New England Journal of Medicine, 349, 1369-1377. https://doi.org/10.1056/NEJMcpc030013

[8] Ban-Hoefen, M., Zeglin, M.A. and Bisognano, J.D. (2008) Diffuse Large B Cell Lymphoma Presenting as a Cardiac Mass and Odynophagia. Cardiology Journal, 15, 471-474.

[9] Habertheuer, A., Ehrlich, M., Wiedemann, D., Mora, B., Rath, C. and Kocher, A. (2014) A Rare Case of Primary Cardiac B Cell Lymphoma. Journal of Cardiothoracic Surgery, 9, 14. https://doi.org/10.1186/1749-8090-9-14

[10] Johri, A., Baetz, T., Isotalo, P.A., Nolan, R.L., Sanfilippo, A.J. and Ropchan, G. (2009) Primary Cardiac Diffuse Large B Cell Lymphoma Presenting with Superior Vena Cava Syndrome. Canadian Journal of Cardiology, 25, e210-e212. https://doi.org/10.1016/S0828-282X(09)70110-2

[11] Jonavicius, K., Salcius, K., Meskauskas, R., Valeviciene, N., Tarutis, V. and Sirvydis, V. (2015) Primary Cardiac Lymphoma: Two Cases and a Review of Literature. Journal of Cardiothoracic Surgery, 10, 138. https://doi.org/10.1186/s13019-015-0348-0

[12] Hans, C.P., Weisenburger, D.D., Greiner, T.C., Gascoyne, R.D., Delabie, J., Ott, G., et al. (2004) Confirmation of the Molecular Classification of Diffuse Large B-Cell Lymphoma by Immunohistochemistry Using a Tissue Microarray. Blood, 103, 275-282. https://doi.org/10.1182/blood-2003-05-1545

[13] Cioc, A.M., Jessurun, J., Vercellotti, G.M. and Pambuccian, S.E. (2014) De Novo CD5-Positive Primary Cardiac Diffuse Large B-Cell Lymphoma Diagnosed by Pleural Fluid Cytology. Diagnostic Cytopathology, 42, 259-267. https://doi.org/10.1002/dc.22918

[14] Shipp, M.A., Harrington, D.P., Anderson, J.R., Armitage, J.O., Bonadonna, G., Brittinger, G., et al. (1993) A Predictive Model for Aggressive Non-Hodgkin's Lymphoma. New England Journal of Medicine, 329, 987-994. https://doi.org/10.1056/NEJM199309303291402

[15] Krol, A.D., le Cessie, S., Snijder, S., Kluin-Nelemans, J.C., Kluin, P.M. and Noordijk, E.M. (2003) Primary Extranodal Non-Hodgkin's Lymphoma (NHL): The Impact of Alternative Definitions Tested in the Comprehensive Cancer Centre West Population-Based NHL Registry. Annals of Oncology, 14, 131-139. https://doi.org/10.1093/annonc/mdg004

[16] Muller, A.M., Ihorst, G., Mertelsmann, R. and Engelhardt, M. (2005) Epidemiology of Non-Hodgkin's Lymphoma (NHL): Trends, Geographic Distribution, and Etiology. Annals of Hematology, 84, 1-12. https://doi.org/10.1007/s00277-004-0939-7

[17] McDonnell, P.J., Mann, R.B. and Bulkley, B.H. (1982) Involvement of the Heart by Malignant Lymphoma: A Clinicopathologic Study. Cancer, 49, 944-951. https://doi.org/10.1002/1097-0142(19820301)49:5<944::AID-CNCR2820490519>3.0 .CO;2-C

[18] Farfan-Leal, F., Esteban, A., Hinojar, R., Garcia-Cosio, M. and Contreras, F. (2019) Primary Cardiac Natural Killer/T-Cell Lymphoma, a Very Rare Form of Lymphoma. Asian Cardiovascular \& Thoracic Annals, 27, 210-212. https://doi.org/10.1177/0218492318798230

[19] Van Rooijen, C.R., Scholtens, A.M., de Jong, C.N., Saraber, C.E. and van de Donk, 
N. (2017) Primary Cardiac Lymphoma with Central Nervous System Relapse. Clinical Case Reports, 5, 1454-1458. https://doi.org/10.1002/ccr3.1094

[20] Antoniades, L., Eftychiou, C., Petrou, P.M., Bagatzounis, A. and Minas, M. (2009) Primary Cardiac Lymphoma: Case Report and Brief Review of the Literature. Echocardiography, 26, 214-219. https://doi.org/10.1111/j.1540-8175.2008.00757.x

[21] Liu, X., Liu, P. and Li, J. (2015) EBV+ Diffuse Large B-Cell Lymphoma Arising within Atrial Myxoma in Chinese Immunocompetent Patient. International Journal of Clinical and Experimental Pathology, 8, 2159-2164.

[22] d'Amati, G., di Gioia, C.R. and Gallo, P. (2001) Pathological Findings of HIV-Associated Cardiovascular Disease. Annals of the New York Academy of Sciences, 946, 23-45. https://doi.org/10.1111/j.1749-6632.2001.tb03901.x

[23] Alam, M., Higgins, R., Alam, Z., Janakiraman, N. and Gorman, M. (1998) Aspergillus Fungal Mass Detected by Transesophageal Echocardiography. Journal of the American Society of Echocardiography, 11, 83-85. https://doi.org/10.1016/S0894-7317(98)70127-0

[24] Berarducci, L., Ford, K., Olenick, S. and Devries, S. (1993) Invasive Intracardiac Aspergillosis with Widespread Embolization. Journal of the American Society of Echocardiography, 6, 539-542. https://doi.org/10.1016/S0894-7317(14)80474-4

[25] McAllister, H.A., Hall, R.J. and Cooley, D.A. (1999) Tumors of the Heart and Pericardium. Current Problems in Cardiology, 24, 57-116.

https://doi.org/10.1016/S0146-2806(99)80001-2

[26] Reynen, K. (1995) Cardiac Myxomas. New England Journal of Medicine, 333, 1610 1617. https://doi.org/10.1056/NEJM199512143332407

[27] Vandael, E., Vandenberk, B., Vandenberghe, J., Willems, R. and Foulon, V. (2017) Risk Factors for QTc-Prolongation: Systematic Review of the Evidence. International Journal of Clinical Pharmacy, 39, 16-25. https://doi.org/10.1007/s11096-016-0414-2

[28] Annibali, O., Nenna, A., Barbato, R., Chello, M., Sedati, P., Bianchi, A., et al. (2018). Primary Cardiac MYC/BCL6 Double Hit Non-Hodgkin Lymphoma. Journal of Cardiology Cases, 17, 103-106. https://doi.org/10.1016/j.jccase.2017.11.002

[29] Soon, G., Ow, G.W., Chan, H.L., Ng, S.B. and Wang, S. (2016) Primary Cardiac Diffuse Large B-Cell Lymphoma in Immunocompetent Patients: Clinical, Histologic, Immunophenotypic, and Genotypic Features of 3 Cases. Annals of Diagnostic Pathology, 24, 40-46. https://doi.org/10.1016/j.anndiagpath.2016.05.005

[30] Petrich, A.M., Gandhi, M., Jovanovic, B., Castillo, J.J., Rajguru, S., Yang, D.T., et al. (2014) Impact of Induction Regimen and Stem Cell Transplantation on Outcomes in Double-Hit Lymphoma: A Multicenter Retrospective Analysis. Blood, 124, 2354-2361. https://doi.org/10.1182/blood-2014-05-578963

[31] Hyo, R., Tomita, N., Takeuchi, K., Aoshima, T., Fujita, A., Kuwabara, H., et al. (2010) The Therapeutic Effect of Rituximab on CD5-Positive and CD5-Negative Diffuse Large B-Cell Lymphoma. Hematological Oncology, 28, 27-32. https://doi.org/10.1002/hon.896

[32] Petrich, A.P., Cho, S.I. and Billet, H. (2011) Primary Cardiac Lymphoma: An Analysis of Presentation, Treatment, and Outcome Patterns. Cancer, 117, 581-589. https://doi.org/10.1002/cncr.25444 